\title{
Strength and Plasticity of H- and Oxide- Terminated Cubic Si Nanocrystals
}

\author{
Andrew J. Wagner ${ }^{1}$, Eric Hintsala ${ }^{1}$, Uwe Kortshagen ${ }^{2}$, William Gerberich ${ }^{1}$ and K. A. Mkhoyan ${ }^{1}$ \\ 1. Department of Chemical Engineering \& Materials Science, University of Minnesota, Minneapolis, \\ $\mathrm{MN}$ \\ 2. Department of Mechanical Engineering, University of Minnesota, Minneapolis, MN
}

Greater understanding of the structure-property relationships contributing to exceptional strength and plasticity in nanoscale materials is vital for the continued miniaturization of electron and MEMS devices [1]. Recent innovations have enabled in-situ investigations of mechanical properties in the TEM at a size scale where a material's defect density can approach zero, allowing intrinsic material properties to be studied. Silicon, being a ubiquitous component of micro-devices, has been studied extensively via ex-situ [2,3] and in-situ [4,5] mechanical testing. However, direct observation of the dynamic processes under deformation has not been scaled beyond 50 to $100 \mathrm{~nm}$. Here, we investigate the elastic-plastic response of 25 to $40 \mathrm{~nm}$ cubic Si nanocrystals (NCs) under [001] compression.

Dislocation-free cubic Si NCs, 25 to $40 \mathrm{~nm}$ in size and having [001] facets with slight asperity [6,7] were synthesized in a flow-through plasma reactor and deposited directly onto sapphire wedges. NCs were compressed at a strain rate of $10^{-2} \mathrm{~s}^{-1}$ by a flat diamond tip of a Hysitron PI95 Picolndenter modified for improved lateral stability. ZLP-filtered images were acquired using the tv-rate CCD of a Gatan GIF 2002 attached to an FEI Tecnai F30 (S)TEM operating at $200 \mathrm{keV}$. Specimens transferred quickly to the TEM remained oxide-free while delays allowed a 1-2 nm oxide to form, enabling H-terminated and oxide-passivated NCs to be studied. To provide contrast of the $\{111\}<1 \overline{1} 0>$ slip system, the NC was rotated for view along its [100] or [110] zone axis. Displacement and contact width were measured in-situ and fitted assuming a circular contact area to determine the true stress and strain.

Stress-strain relationships, Fig. 1a, were largely independent of NC size and for clarity were fitted as an average of all compressions, Fig. 1b. Large linear elastic response was observed with strains of $10 \%$ and yield strengths up to 8.5 and $9.5 \mathrm{GPa}$ for $\mathrm{H}$-terminated and $\mathrm{SiO}_{2}$-covered $\mathrm{NCs}$, respectively. The loading curve of $\mathrm{SiO}_{2}$-capped NCs demonstrated a kink between $10 \%$ and $15 \%$ strain, point $\mathrm{A}$ in Fig. $1 \mathrm{~b}$. While compressing a $39 \mathrm{~nm} \mathrm{NC}$ viewed along the [110] direction, Fig. 2a, broad strain contours were seen to expand from the point of contact, Fig $2 \mathrm{~b}$. At point A, sharp ripples of contrast were observed adjacent to the indenter contact surface, Fig 2c. Additional strain resulted in motion of these bands, believed to be prismatic loops, away from the stress concentration. A load drop occurred at $20 \%$ strain, point B, associated with the formation of sharp slip bands originating at the center of the $\mathrm{NC}$ and extending along the $\{111\}$ planes to the NC corner, Fig. $2 \mathrm{~d}$. The upper yield point was followed by a period of softening attributed to the ability of dislocations to escape through the NC corners, owing to their slight truncation. As the strain was increased beyond Point $\mathrm{C}$, the corners became constrained by the sapphire, trapping dislocations and building a back stress that contributed to work hardening, Fig. $2 \mathrm{e}$.

Work is ongoing to interpret post-mortem HR-TEM images and CBED patterns to better understand the deformation mechanisms. In-situ recordings of slip bands growing across the NC suggest dislocation velocity can be measured directly. Load-controlled compressions are also being performed to examine residual strain and reverse plasticity and to better understand the strengthening role of the native oxide. 


\section{References:}

[1] R. Suk-Kyu et al, Device and Materials Reliability, IEEE Transactions on 11 (2011) p. 35.

[2] M. J. Cordill et al, Acta Mater. 54 (2006) p. 4515.

[3] W. W. Gerberich et al, Journal of the Mechanics and Physics of Solids 51 (2003) p. 979.

[4] D. D. Stauffer et al, Acta Mater. 60 (2012) p. 2471.

[5] J. Deneen et al, Journal of Materials Science 41 (2006) p. 4477.

[6] A. Bapat et al, Plasma Phys. Controll. Fus. 46 (2004) p. 97.

[7] A. J. Wagner et al, Nano Lett. 13 (2013) p. 5735.

[8] This work was supported by the MRSEC Program of the NSF under DMR-0819885. Parts of this work were carried out in the Characterization Facility, University of Minnesota.

(a)

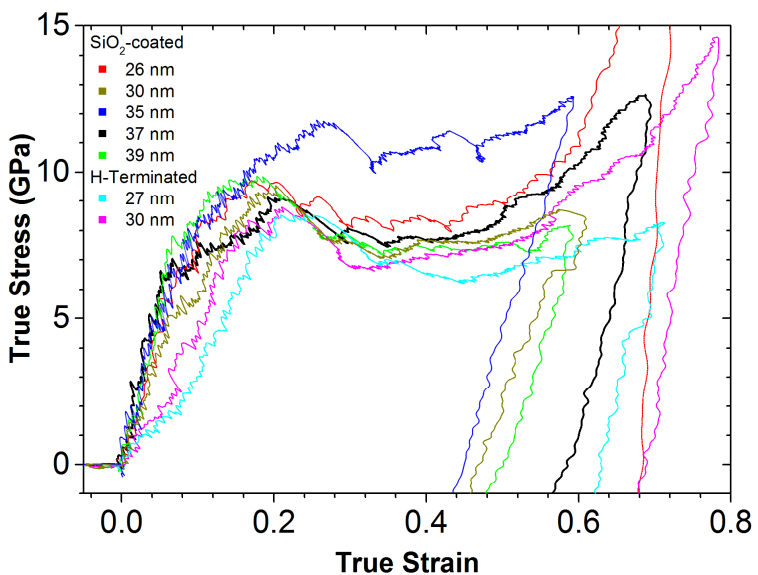

(b)

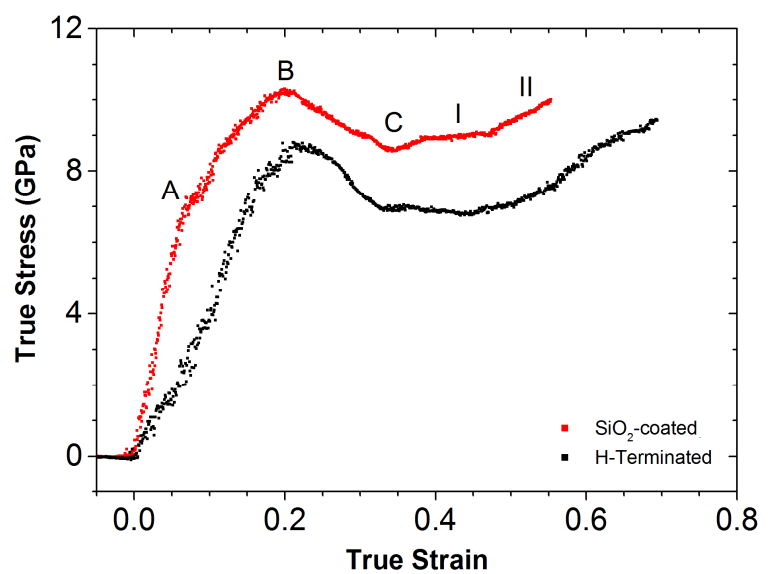

Figure 1. (a) Stress-strain plots for cubic Si NCs under [001] compression. (b) Averaged stress-strain plots for H-terminated and $\mathrm{SiO}_{2}$-terminated $\mathrm{Si}$ NCs. Oxide-capped NCs exhibit increased strength, increasing from $8.5 \mathrm{GPa}$ to $9.6 \mathrm{GPa}$, while elastic strains as high as $10 \%$ are obtained. Point $\mathrm{A}$ is attributed to nucleation of prismatic loops. Point B is associated with initiation of $\{111\}<110>$ slip as dislocations escape out the unconstrained corners of the $\mathrm{NC}$, resulting in a load drop to point $\mathrm{C}$ and Stage I hardening. As the corners become constrained, pileup leads to Stage II hardening.
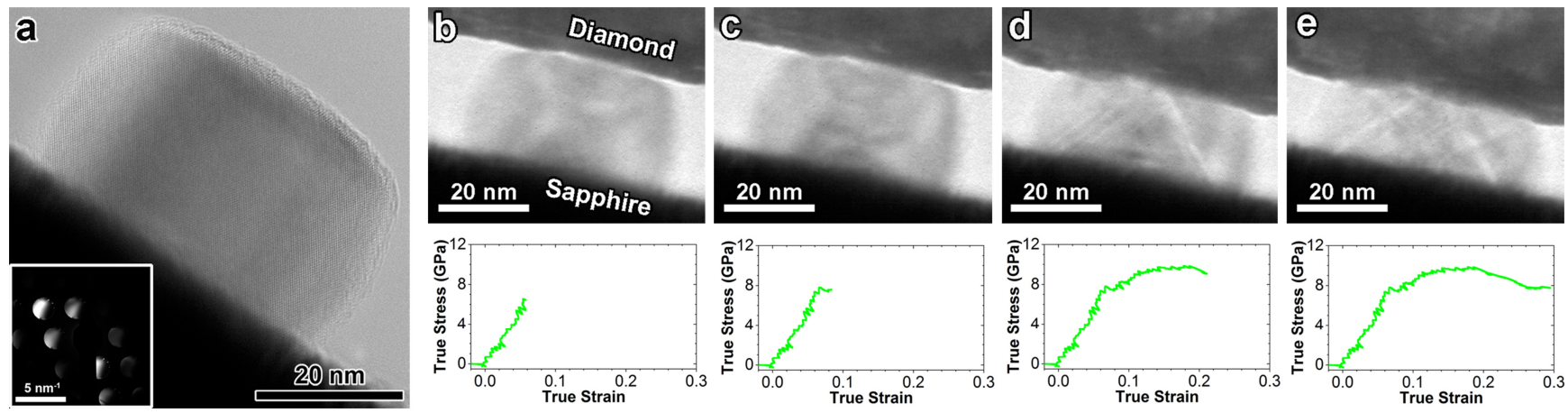

Figure 2. Displacement-controlled compression of $39 \mathrm{~nm} \mathrm{Si} \mathrm{NC} \mathrm{capped} \mathrm{with} \mathrm{native} \mathrm{oxide,} \mathrm{viewed}$ along its [110] direction. (a) HR-TEM/CBED image of the original NC. (b-e) The particle is compressed elastically (b) until about $10 \%$ strain (c) after which small dislocation loops form at the stress concentration at the indenter contact. (d) Slip initiates at around $20 \%$ strain. (e) As the NC is compressed further, the asperity is eliminated and dislocation pile up leads to the observed work hardening. (f) Post-mortem TEM/CBED image of NC. 